Received $25^{\text {th }}$ August 2020, Accepted $16^{\text {th }}$ September 2020

Link to DOI:

10.25220/WNJ.V04.S2.0002

Journal Website:

www.worldnutrijournal.org

\title{
Caesarean section and gut microbiota in children
}

\author{
Ariani D Widodo ${ }^{1}$, Mohammad Juffrie ${ }^{2}$ \\ 1. Pediatric Gastrohepatology Working Group, Harapan Kita National Center for Woman and \\ Child Health Care, Jakarta, Indonesia \\ 2. Pediatric Department, Medical Faculty, Gadjah Mada University-Sardjito Hospital, Yogyakarta, \\ Indonesia
}

\begin{abstract}
Over the last two decades, the C-section rate has increased worldwide. It is understood that colonization patterns of intestinal microbiota in infant delivery in C-section vary from those that were delivered vaginally. These different microbial pattern and diversity will impact and respond to immune and dysbiosis-related diseases. This article examined the effect of $\mathrm{C}$-section on gut microbiota in children.

Recent findings: Newborns are influenced by various factors, including mode of delivery, feeding, nutrition, hospitalization, antibiotic and host gene. Several studies have shown that infants with Csection have lower Bifidobacterium while others have shown lower abundance of Enterobactericeae and Bacteroides in infants with C-section compared to infants born vaginally. Although the mode of delivery is only one factor that influences infant microbiota composition, studies conclude that reduced microbial exposure during the $\mathrm{C}$-section is important because it can affect dysbiosis several years after birth. Good microbiota is a key source of microbial-driven immune regulation, changes in normal patterns of bacterial colonization can alter the immune development outcome and may predispose to certain immune-related disorders later in life. Summary: The composition and concentrations of intestinal microbiota between vaginally and Csection born infants are significantly different. Among C-section infants, gut microbiota is associated with lower diversity and therefore induces dysbiosis, which can affect immune development and may predispose to some immune disorders, i.e. allergies in particular. Nutritional approach with pre-, probiotics, and/or synbiotics can have a promising effect early in life in preventing gut dysbiosis.

Keywords: Caesarean section, children, gut microbiota, dysbiosis, synbiotic, probiotic, prebiotic
\end{abstract}

\section{Introduction}

The term 'microbiota' refers to the number of all microbial colonies living in or on the human body. The human gut, primarily the large intestine,

\begin{tabular}{l}
\hline Corresponding author: \\
Dr.dr. Ariani D. Widodo, SpA(K) \\
Pediatric Gastrohepatology Working Group, \\
Harapan Kita National Center for Woman and Child \\
Health Care, Jakara, Indonesia \\
Email: dr.ariani@gmail.com \\
\hline
\end{tabular}

contains $10^{14}$ bacteria, the body's largest numbers of microbiota compared to certain human bodies such as skin, vagina, mouth and ears. Gut microbiota coexists with host in a homeostatic relationship and has bidirectional interaction with the immune system. In the first year of life, the production of gut microbiota is a complex process and an important process in the human life cycle as it plays a key role in immunological and metabolic processes, thereby affecting human health and diseases. ${ }^{1}$

The pioneering bacteria colonizing infant intestine and its shift towards a stable ecosystem are crucial to the development of optimal interaction 
(symbiosis). ${ }^{2}$ Tissier published the idea in 1900 that fetuses are sterile in utero and microbial colonization begins later during and after birth, this is recognized for centuries. In comparison, studies have shown that uterine microbiota is associated with healthy term pregnancies and there is potential for placental barrier bacterial transmission. Recent studies have detected bacteria from healthy newborns in placenta tissue, umbilical cord blood, amniotic fluid, meconium and fetal membranes with no sign of infection or inflammation..$^{2,3}$

The human bowel is easily colonized by a large variety of microbes and is distinctive in composition, but over $95 \%$ can be allocated to four main phylas: Firmicutes, Bacteroidetes, Actinobacteria and Proteobacteria. ${ }^{3}$ The formation of microbiota starts at birth and plays an important role in host immune system maturation, defending against pathogens and providing nutrients. ${ }^{4}$ Influenced by many factors, the diversity and colonization patterns of infant gut microbiota include pregnancy, mode of delivery (vaginal vs Caesarean section), diet (breast milk vs formula), sanitation, and antibiotic treatment. ${ }^{5}$

The newborns' first big exposure to microbes happens during birth and significantly influenced by delivery methods. ${ }^{3}$ The skin, gut, oral and nasopharyngeal cavities of the vaginally-born neonates are initially seeded by Lactobacillus spp, which resembles maternal vaginal microbiota. The absence of Lactobacillus spp and dominance of specific skin and environmental microbes such as Staphylococcus, Streptococcus or Propionibacteria is found in infants delivered by Caesarean section (C-section). ${ }^{6,7}$ These initial microbiota evolve over time and are adapting to the body and formed by the availability of different nutrients. Recent study showed that although the difference between delivery modes gradually decreased at 4 months and 12 months of age, C-section-born infants remained have more heterogeneous gut microbiota compared to the vaginally-born. ${ }^{3}$

The rate of $\mathrm{C}$-section has been increasing worldwide over the last two decades. It is observed that intestinal colonization patterns of infant delivery in $\mathrm{C}$-section vary from those that were vaginally delivered. However, the relationship between these trends and delivery mode is still unclear. Various colonization patterns and diversity of the intestinal microbiota can impact and respond to immune development and also with several illnesses associated with changes of the intestinal microbiota (dysbiosis) ${ }^{8}$ Thus, this article analyzes the relationship of Caesarean section (C-section) delivery and its effect on the gut microbiota of children.

\section{Methods}

This article reviewed how C-Section affect gut microbiota and its effect on children. We focus on gut microbiota, the difference between different delivery method on gut microbiota, and how it affect children's health. Articles included in this review are from several electronic database i.e Pubmed, Medline and Google Scholar with the key words "mode of delivey", "ceasarean section", "gut microbiota", "dybiosis", "prebiotics", "probiotics" and "synbiotics". Articles included are the one in English or Indonesia language and published from April 2010 until April 2020.

\section{Results and Discussion}

\section{Gut microbiota within the first 100 days of life}

The sterility of uterus and microbial colonization of newborn is generally recognized since 1900 . This view is based on the fact that microbial analyses in pregnancy-related samples were conducted only in cases of suspected infection. It is also discussed as culture studies have shown that microbes are found in placenta, amniotic fluid, fetal membrane, umbilical cord blood and meconium. ${ }^{2}$

Nearly half of the umbilical cord samples and all of the meconium samples contain bacteria from cultivable sources such as Enterococcus, Streptococcus, Staphylococcus and Propionibacterium, as well as human placental bacteria Bifidobacterium and Lactobacillus. ${ }^{4}$ The mechanism by which gut bacteria enter human uterus is not fully understood. Several studies have shown that certain bacteria from the maternal digestive tract can spread in healthy host to extra digestive locations. The mechanism consists of dendritic cells and $\mathrm{CD} 18^{+}$cells that may take nonpathogenic bacteria from the intestinal lumen and transport them to other locations. ${ }^{9}$ However, 
dendritic cells may open tight intersections between intestinal epithelial cells, send dendrites out of the epithelium and directly sample bacteria without damaging the epithelial barrier. It has also been shown that small numbers of dendritic intestinal cells can maintain live commensal bacteria in mesenteric lymph nodes for several days. Dendritic cells and/or macrophages, once within the lymph nodes, can spread elsewhere because lymphocytes circulate in the associated lymphoid system. ${ }^{9,10}$ This hypothesis is confirmed by a study which found Enterococcus faecium in amniotic fluid and meconium following sterile $\mathrm{C}$-section of pregnant mice previously inoculated with the bacteria. ${ }^{2}$ This could be transferred to the fetus via the bloodstream. A recent research has also found that microbiota is present in human placenta and has shown that it can be the route for maternal oral bacteria to be given to fetus like microbiota in the oral cavity. ${ }^{11}$ The microbial interaction in prenatal life is thus seen, but previous studies have not used techniques to quantify the number of bacterial cells and to differentiate accurately between DNA, dead cells, living cells and active cells that make it difficult to identify placental microbiota or pregnancy risk factors. ${ }^{11}$ It requires further research to validate the presence, amount and effect of intrauterine microbiota on the health of children.

Post-natal microbial interaction is higher and extensive during birth and breastfeeding than in prenatal life. Newborns' first exposure to microbes happens during the birth process and may differ according to delivery method. Lactobacillus spp is dominant in vaginally-delivered newborns but different species are present in each newborn because of various motherly vaginal microbiota. Newborns therefore continue to live exposed to various bacterial species, depending on interaction with vaginal flora. In comparison, $\mathrm{C}$-section born children showed various Staphylococcus, Streptococcus, Clostridium or Propionibacterium colonization. This indicates that early colonization benefits bacterial communities associated with each mode of delivery. ${ }^{6,12}$

In newborns, gut microbiota has a low diversity and characterized by the relative prevalence of the phyla Proteobacteria and Actinobacteria. The colonization begins first with optional anaerobic species, followed by the creation of compulsory anaerobes organisms such as Bifidobacterium, Bacteroides and Clostridium. Full-term breast-fed infants show dominance of compulsory anaerobic bacteria, such as Bifidobacteria in the gut, while premature infants show lower bacteria but lower facultative anaerobes such as Enterobacteriaceae and Enterococcaceae. In addition, the proliferation and dominance of other bacteria, such as Firmicutes and Bacteroidetes diversify the microbiota. Finally, by the end of the first year of life, children microbial profile start to resemble that in adult, and by 2-3 years of life have developed their signature microbial profile..$^{3,13}$

Newborn microbiota is a complex ecosystem that is influenced by various factors such as delivery mode, feeding/dieting, medication, hospitalization and host gene. Demographic factors such as gender, gestational age and postnatal age also contribute in the development of gut microbiota. These contributing factors have been identified in several studies, and most have shown that interaction between host-microbial products and their different factors play an important role in infant health and immune development processes. ${ }^{13,14}$

\section{Impact of C-section and gut dysbiosis in children}

There are many factors influencing the production of gut microbiota such as delivery mode, diet, genetics, health status, gestational age, etc. Breast milk encourages intestinal microbiota production by adding probiotics and prebiotics and providing protection to pathogens. Lactobacillus, Bifidobacterium, Staphylococcus, Bacteroides, Enterococcus, Streptococcus and Clostridium are several examples of breast milk bacteria. These bacteria are evident in breast milk and neonatal feces, which indicate vertical transmission of bacteria through breastfeeding. Full-term infants that are exclusively breastfed tend to have more Bifidobacterium, but less diverse gut microbiota compared to formula-fed infants. When children are exposed to solid food, the gut microbiota pattern shifts towards adult microbiota pattern at around 12 months of age. ${ }^{13}$

The use of antibiotics will alter the microbial diversity and affect the health of children. Shortterm use of antibiotics will significantly affect the colonization and diversity of gut microbiota. Good 
microbiota diversity has been shown to decrease significantly in the first three weeks of life in infants with longer-term antibiotics treatment, compared with infants exposed to short-term antibiotics. In infants, it is shown that after antibiotic treatment, the colonization pattern of Bifidobacterium decreases while Proteobacteria increases. Preterm infants are often delivered through $\mathrm{C}$-section and the use of antibiotics in this situation is common. In this case, the evolving pattern of microbiota may be associated with a serious intestinal disorder, such as neonatal necrotizing enterocolitis (NEC). ${ }^{15}$

Initial microbial exposure occurs during and shortly after birth, as described. The newborn's first high exposure to microbes occurs during childbirth and relies heavily on mode of delivery. ${ }^{13}$ In infants born vaginally and via the $\mathrm{C}$-section, different bacterial colonization occurs. Vaginal bacteria are transmitted from mother to child during vaginal birth, primarily Lactobacilli, while infants born with C-section have microbial pattern resembling environmental microbes. ${ }^{6,8,12}$ It is known that in infants born with C-section, the gut microbiota in the first week of life is less diversed compared with those delivered vaginally. Nevertheless, the pattern of colonization of its phylum is not affected by the mode of delivery. Some studies have shown lower Bifidobacterium and Enterobactericeae in C-section infants compared with those delivered vaginally. Meanwhile, Clostridium genus is more common in $\mathrm{C}$-section delivered infants. Four studies have indicated that the Bacteroides phylum was less diversed among infants delivered in C-section within the first week, and two studies have shown that there is no significant difference within the Firmicute phylum between C-section and vaginallydelivered infants. ${ }^{8}$

$\mathrm{C}$-section has saved many lives but has become overperformed, often with no or weak indications, over the last decades. In creating "good" gut microbiota profile, the existence of healthy/abundant diversity plays a decisive role. Increased C-section rate, prematurity, antibiotics usage, improvements in feeding and the environment, may have affected gut microbiota. The transformation of microbial communities at risk of dysbiosis (microbial imbalance). Microbiota plays a key role via symbiosis in humans (controllable benefits), which may lead to short-term inflammation that leads to children's gastrointestinal disorders. Several studies have shown that dysbiosis of children coincides with emerging immune changes (asthma), inflammatory bowel disease and metabolic (obesity) disorders. ${ }^{16}$

A balanced relationship between the host microbiota and the immune system is essential for a homeostatic toward pathogenic attack and avoid inflammation. Infants with dysbiosis gut foster a strong T-helper (Th)-1 bias, which makes the immune system susceptible to inflammation hence secreting cytokines Interleukin (IL)-12 and Interferon (IFN)- $\gamma$. This inflammation affects tissue and tissue repair, disturbing the normal immune system, potentially leading to long-term consequences such as inflammatory bowel disease (IBD), allergy and autoimmune disease. ${ }^{3}$

C-section born infants have a lower diversity of microbiota compared with vaginally born infants, especially on Bacteroides colonization. B. fragilis which significantly higher in infants born vaginally, has been shown to have an anti-inflammatory role by acting on $\mathrm{T}$ cells. B. fragilis produces surface polysaccharide A (PSA), a microorganismassociated molecular pattern (MAMP) that is recognized by toll-like receptor 2 (TLR 2) on T cells. Engagement of TLR2 and PSA leads to T cell induction and limits Th-17 response which will promote tolerance and immunosuppression in the gut. ${ }^{3}$ Appropriate microbial stimulation during infancy is required to develop Th1-like and T-cell response. Difference in infants gut microbiota could shape later immune responsiveness, influencing Th1 maturation may impact on immune mediated disease. ${ }^{17}$ Lower microbial diversity might explain why infants born by $\mathrm{C}$-section are associated with the development of allergy and asthma, type-1 diabetes, and obesity. $3,14,17$

\section{Gut dysbiosis and its impact on health}

Inadequate transfer of maternal microbiome to infants born through $\mathrm{C}$-section may lead to impaired immunological development. Although mode of delivery is only one factor that influences the composition of infant microbiota, studies conclude that reduced microbial exposure during $\mathrm{C}$-section is important because it may cause dysbiosis several years after birth. Gut microbiota is an important key 
source of microbial-driven immune regulation. Alterations of the normal bacterial colonization patterns may change the outcome of immune development and may predispose to certain immune-related disorders later in life, such as allergy, obesity or diabetes. ${ }^{18}$

The development of the newborn immune system depends on the gut microbiota composition in early life. Hypothesis on microbial deprivation syndrome in economically-developed countries and its association with high incidence of multiple sclerosis, type-1 diabetes and Crohn's disease has been proposed. ${ }^{19}$ Low-diversity microbiota leads to insufficient T-cell induction with regulatory and/or Th1-like properties to counteract $\mathrm{Th} 2$ response in induced allergies. Early exposure to diverse gut microbiota is important as the distribution of specific microbial species in immune maturation. Repeated exposure to new microbial antigens may enhance the development of immune response through Th1-like responses. ${ }^{17}$ A study using cultureindependent bacterial molecular techniques concludes low gut microbiota diversity during childhood in children with eczema or sensitization. ${ }^{14}$ Indeed, a number of cross-sectional epidemiological studies showed differences in the composition and activity of gut microbiota among healthy and atopic children. Asthma incidence was also linked to abnormal microbiota in pediatric populations. ${ }^{19} \mathrm{~A}$ study reported that gut microbiota depletion at 3 months of age was found in subjects with atopy and wheeze. In this group, biosynthesis of lipopolysaccharides and short-chain fatty acid (SCFA) acetate is also reduced. ${ }^{3}$

Gut microbiota also contributes to the production of SCFA in fat tissues to the formation of triglycerides block through fermentation of indigestible polysaccharide-derived plants. Kalliomaki ${ }^{20}$ found lower intestinal Bifidobacterium and higher concentration of Staphylococcus aureus at 6 and 12 months in obese children compared to children with normal weight. Alterations in early exposures to microbiota (such as C-section delivery), lack of breastfeeding, maternal pregnancy BMI, and infants antibiotics, may alter the diversity of microbiota and may influence the risk of overweight in later childhood.

Aberrant gut microbiota colonization can affect the immune system in adulthood, making it more susceptible to certain diseases. Persistent increases in natural killer cells can only be prevented by neonatal colonization of the microbiota, and microbiota also drives the generation of $\mathrm{T}$ cell that control inflammation. IgA secretion and inflammatory cytokine profiles are also influenced by gut microbiota. This conclude that immune disturbance may be caused by altered gut microbiota, which explains the increased risk of autoimmune disorders in adulthood. ${ }^{18}$

\section{C-section, gut dysbiosis and allergies}

The most common chronic disease in childhood is allergic diseases. Allergic disease is an immunemediated disease that has increased considerably over the past decades with a high increase in Csection deliveries. ${ }^{2}$ There are reports that there is a risk of developing allergic disease, allergic rhinitis, asthma and asthma-related hospitalization, and food allergy, whereas there was no association with inhalant atopy and eczema/atopic dermatitis. ${ }^{21,22}$ As already mentioned, gut microbiota plays an important role in improving immune system development and maturation. Gut dysbiosis has been shown to be associated with atopic children in several studies. Allergic infants have been found to have less Bifidobacterium colonization than in nonallergic infants. ${ }^{23}$ Reduced exposure to microbial antigens to the gut explain immune system dysregulation. To develop a more balanced immune phenotype, appropriate microbial stimulation during childhood, including maturation of Th1-like responses and appropriate development of regulatory $\mathrm{T}$-cell response is required. The imbalance in gut microbiota has affected the Th1 ripening pathway showing that children born through C-section have lower levels of Th-1 chemokines CXCL 10 and CXCL $11 .{ }^{17}$ A study by Kalliomaki first showed the difference between Bifidobacteria and Clostridia in newborns. Diversed and balanced gut microbiota are important for normal functioning of the immune system. ${ }^{23}$ Other study also showed that in term of gut microbiota composition, nutritional intervention might also play a role on gut microbiota development and showed specific benefit including economic effectiveness. ${ }^{24}$ 
C-Section delivery is a risk factor for the development of allergies and has been linked to reduced relative abundance and diversity of Bacteroides in first year of life. ${ }^{17,25,26}$ High Bifidobacterium content is considered to lead to better immune system development and maturation. ${ }^{2}$ A meta-analysis shows that $\mathrm{C}$-section delivery has a lower diversity of gut microbiota during the first 3 months of life. At the colonization level, Bifidobacterium and Bacteriodes genera in vaginally delivered infants seem to be significantly more frequent compared to $\mathrm{C}$-section delivered infants. ${ }^{8}$ The presence of Bacteroides was associated with high levels of chemokines associated with Th1 in infancy at 1 month. C-section is associated with lower bacterial diversity as described above and lower circulating levels of chemokines are associated with Th1 in infancy. ${ }^{17}$ Moreover, Ly et al. showed that C-Section delivered infants are characterized by increased cytokine IL-13 production from stimulated cord blood cells. ${ }^{26}$ This suggests higher incidence and severity of asthma and atopy caused by gut dysbiosis in these C-section infants.

\section{Intervention of gut dysbiosis with synbiotics}

The importance of intestinal composition in health, especially during early life, suggests that microbial interventions may be an effective strategy for potential adverse health outcomes. Some possible strategies have been employed in the effort to achieve a healthy balance of good intestinal microbiota such as vaginal seeding, environmental exposure, and supplementation. ${ }^{27}$ The supplementation of prebiotics and/or probiotics is widely studied. Probiotics are live microorganisms that provide hosts with sufficient health benefits when administered. The mechanism of these probiotics' positive effects remains unclear. Prebiotics is a non-digestible nutritional fiber, which has a positive physiological effect for the host by selectively stimulating a small number of indigenous bacteria to increase their growth or activity. Prebiotics is known to boost the growth of beneficial bacteria Lactobacillus and Bifidobacteria in the upper gastrointestinal tract. Prebiotics produce short fatty acids, lactic acids and acetic acids in such bacteria, which improve host metabolism, the immune system and the gastrointestinal function. The immune and metabolic systems combined with special prebiotics and probiotics (called synbiotics) supplements is thought to have synergistic beneficial effects. $^{28}$

Breast milk, as the gold standard for infant nutrition, not only provides nutrients to the infant but is also a source of probiotics (microbiota) and prebiotics (HMOs) contributing to the establishment of the ideal infant gut microbiota. ${ }^{29}$ While breastfeeding, each milliliter of human milk introduces about $10^{3}$ to $10^{6}$ bacterial cells into the body. ${ }^{30,31}$ Breast milk microbiota is dominated by Staphylococcus, Streptococcus, Propionibacterium and Bifidobacterium. The transfer of these microorganisms into the neonatal gut is thought to protect against infections and contribute to the maturation of the immune system. ${ }^{30-32}$ Human milk also contains up to $15 \mathrm{~g} / \mathrm{L}$ of oligosaccharides, whereas only trace amounts are usually found in cow's milk. These human milk oligosaccharides (HMO) act as growth substrates for beneficial gut microbiota such as Bifidobacteria. ${ }^{33}$

Nutritional intervention with pre-, probiotics and/or synbiotics may be a cost-effective approach to early life dysbiosis and its correlation with allergic conditions. Evidence of prebiotic supplementation of infant formula with a specific mixture of short-chain galacto-oligosaccharides (scGOS) and long-chain fructo-oligosaccharides (lcFOS) resulting in an intestinal microbiota enriched with Bifidobacterium. ${ }^{27}$ Knol et al ${ }^{34}$ research has shown that adding $0.8 \mathrm{~g} / 100 \mathrm{ml}$ of prebiotic scGOS/lcFOS in the infant formula enhances the bifidobacterial growth and changes in small, breast-fed infant fatty acids, lactate and $\mathrm{pH}$. Moreover, a randomized clinical trial demonstrated that administration of this specific prebiotic mixture early in life continues to be protective against eczema development at 6 months and even against allergic events up to 5 years, in healthy high-risk infants based on family history of allergy. ${ }^{35-37}$

Existing publications show that intestinal microbiota is typically low in Bifidobacteria and Lactobacilla compared to healthy infants in infants with allergic conditions. ${ }^{27}$ Several studies have shown conflicting results of the presence of Bifidobacteria and their relationship to lower risk for developing allergic diseases. Ismail and 
colleagues assessed the association between various species of Bifidobacterium and subsequent eczema and atopic sensitization. Bifidobacterium breve at 1 week and 3 months of age were shown to be associated with a lower risk of eczema development and sensitization at 12 months of age. ${ }^{38}$ Probiotics supplementation intervention together with extensively hydrolyzed casein formula resulted in cow's milk protein tolerance that reduced incidence of allergic manifestations, including eczema and asthma, in children with cow's milk allergy. In other research synbiotics provide supporting evidence in reducing the risk of symptoms of asthma and the use of asthma medication in children who receive synbiotics with extensively hydrolyzed formula (eHF). These infants also have higher Bifidobacterium and lower Clostridium levels. ${ }^{27}$

Research shows the retarded colonization of Bifidobacterium spp in infants born through Csection by modifying gut microbiota with synbiotics supplement. Early supplementation with scGOS/lcFOS and Bifidobacterium breve (B. breve) $\mathrm{M}-16 \mathrm{~V}$ (synbiotics) led to immediate Bifidobacterium colonization, which suggests that the first three months of the life represent an opportunity for quick recovery. ${ }^{27}$ One study showed protective effect in dysbiosis of mice with food allergy treated with $B$. breve $\mathrm{M} 16-\mathrm{V} .{ }^{39}$ A recent study has shown that $B$. breve abundance was correlated with protection from development of eczema and childhood immune sensitivity. ${ }^{38}$ Chua et al' ${ }^{25}$ pioneering evidence has shown that supplementation with scGOS/lcFOS and $B$. breve $\mathrm{M} 16-\mathrm{V}$ in $\mathrm{C}$-section-born infants allows rapid colonization of bifidobacteria from the first days of life and offers promising nutritional intervention in dysbiosis. In this study, synbiotic mixture was able to re-establish the delayed colonization in elective $\mathrm{C}$-section delivered infants from the first days of life, and remained significant until 2 months of age. Synbiotics created a favorable gut ecosystem milieu that contributes in preventing colonization of fastgrowing opportunistic pathogens and potentially reduce the development of skin disorders, especially eczema in early life in C-section delivered infants.

World Allergy Organization (WAO) recommends using probiotics to children at high risk of developing allergy, and also provided conditional recommendation for prebiotic supplementation in non-exclusively breastfed infants regardless of their allergic risk. ${ }^{25,40,41}$ Although it wasn't supported with a strong certainty of evidence, prebiotic supplementation may place a relatively higher value on possible prevention of allergies and a relatively lower value on additional cost of prebiotic supplementation in not exclusively breastfed infants. ${ }^{41}$ The benefits of human milk oligosacharides (HMOs) intervention were also widely studied and considered safe for infant nutrition, including for gut microflora development of C-section born babies. ${ }^{42}$ Thus the use of prebiotics on top of probiotics, or in a form of synbiotics, in early life may be beneficial to compensate for intestinal microbiota disorders, but more study is needed on the benefits of patient outcomes, particularly in terms of allergic outcomes.

\section{Conclusion}

The intestinal microbiota is a very complex entity and there are a lot more to learn about the underlying processes that shapes microbiota development, interactions with the host, and the role of specific microbes in health and disease. The first major infant exposure that affects gut microbiota is during and shortly after birth, especially related to mode of delivery and breastfeeding. There is a significant difference in the diversity and quantities of gut microbiota between infants born through vaginal and $\mathrm{C}$-section delivery. Gut microbiota in C-section infants is less diversed, and therefore causing dysbiosis. Gut microbiota is the main source of microbial control by $\mathrm{T}$ lymphocytes and gut dysbiosis can alter immune developments and predispose to some immune disorders later in life including allergy, atopy, obesity, diabetes, inflammatory bowel diseases and other autoimmune diseases. The use of pre- and probiotics, or in the form of synbiotics, offers a promising benefit for gut dysbiosis especially in C-section born infants as a cost-effective strategy to prevent metabolism and immune disorders. 


\section{Conflict of Interest}

Authors declared no conflict of interest regarding this article.

\section{Open Access}

This article is distributed under the terms of the Creative Commons Attribution 4.0 International Licence

(http://creativecommons.org/licenses/by/4.0/), which permits unrestricted use, distribution, and reproduction in any medium, provided you give appropriate credit to the original author(s) and the source, provide a link to the Creative Commons license, and indicate if changes were made.

\section{Reference}

1. Wopereis H, Oozeer R, Knipping K, Belzer C, Knol $\mathrm{J}$. The first thousand days-intestinal microbiology of early life: establishing a symbiosis. Pediat Allerg Imm-UK. 2014;25(5):428-38.

2. Rodríguez JM, Murphy K, Stanton C, Ross RP, Kober OI, Juge N, et al. The composition of the gut microbiota throughout life, with an emphasis on early life. Microb Ecol Health Dis. 2015;26(1):26050.

3. Tamburini S, Shen N, Wu HC, Clemente JC. The microbiome in early life: implications for health outcomes. Nat Med. 2016;22(7):713.

4. Belkaid Y, Harrison OJ. Homeostatic immunity and the microbiota. Immunity. 2017;46(4):562-76.

5. Marques TM, Wall R, Ross RP, Fitzgerald GF, Ryan CA, Stanton C. Programming infant gut microbiota: influence of dietary and environmental factors. Curr Opin Biotech. 2010;21(2):149-56.

6. Dominguez-Bello MG, Costello EK, Contreras M, Magris M, Hidalgo G, Fierer N, et al. Delivery mode shapes the acquisition and structure of the initial microbiota across multiple body habitats in newborns. PNAS. 2010;107(26):11971-5.

7. Bäckhed F, Roswall J, Peng Y, Feng Q, Jia H, Kovatcheva-Datchary $\mathrm{P}$, et al. Dynamics and stabilization of the human gut microbiome during the first year of life. Cell Host Microbe. 2015;17(5):690703.

8. Rutayisire E, Huang K, Liu Y, Tao F. The mode of delivery affects the diversity and colonization pattern of the gut microbiota during the first year of infants' life: a systematic review. BMC Gastroenterol. 2016;16(1):86.

9. Rescigno M, Urbano M, Valzasina B, Francolini M, Rotta G, Bonasio R, et al. Dendritic cells express tight junction proteins and penetrate gut epithelial monolayers to sample bacteria. Nat Immunol. 2001;2(4):361-7.

10. Fernández L, Langa S, Martín V, Maldonado A, Jiménez E, Martín R, et al. The human milk microbiota: origin and potential roles in health and disease. Pharmacol Res. 2013;69(1):1-10.

11. Aagaard K, Ma J, Antony KM, Ganu R, Petrosino J, Versalovic J. The placenta harbors a unique microbiome. Sci Transl Med. 2014;6(237):237ra65ra65.

12. Dominguez-Bello MG, De Jesus-Laboy KM, Shen N, Cox LM, Amir A, Gonzalez A, et al. Partial restoration of the microbiota of cesarean-born infants via vaginal microbial transfer. Nat Med. 2016;22(3):250.

13. Cong $\mathrm{X}, \mathrm{Xu} \mathrm{W}$, Romisher R, Poveda $\mathrm{S}$, Forte $\mathrm{S}$, Starkweather A, et al. Focus: Microbiome: Gut microbiome and infant health: Brain-gut-microbiota axis and host genetic factors. Yale J Biol Med. 2016;89(3):299.

14. Garcia MCS, Yee AL, Gilbert JA, Dsouza M. Dysbiosis in children born by caesarean section. Ann Nutr Metab. 2018;73(3):24-32.

15. Greenwood C, Morrow AL, Lagomarcino AJ, Altaye M, Taft DH, Yu Z, et al. Early empiric antibiotic use in preterm infants is associated with lower bacterial diversity and higher relative abundance of Enterobacter. Pediatrics. 2014;165(1):23-9.

16. Francavilla R, Cristofori F, Tripaldi ME, Indrio F. Intervention for dysbiosis in children born by $\mathrm{C}$ section. Ann Nutr Metab. 2018;73(3):33-9.

17. Jakobsson HE, Abrahamsson TR, Jenmalm MC, Harris K, Quince C, Jernberg C, et al. Decreased gut microbiota diversity, delayed Bacteroidetes colonisation and reduced Th1 responses in infants delivered by caesarean section. Gut. 2014;63(4):55966.

18. Sandall J, Tribe RM, Avery L, Mola G, Visser GH, Homer CS, et al. Short-term and long-term effects of caesarean section on the health of women and children. Lancet. 2018;392(10155):1349-57.

19. Renz H, Brandtzaeg P, Hornef M. The impact of perinatal immune development on mucosal homeostasis and chronic inflammation. Nat Rev Immunol. 2012;12(1):9-23.

20. Ajslev T, Andersen C, Gamborg M, Sørensen T, Jess T. Childhood overweight after establishment of the gut microbiota: the role of delivery mode, pre- 
pregnancy weight and early administration of antibiotics. Int J Obesity. 2011;35(4):522-9.

21. Van Nimwegen FA, Penders J, Stobberingh EE, Postma DS, Koppelman GH, Kerkhof M, et al. Mode and place of delivery, gastrointestinal microbiota, and their influence on asthma and atopy. J Allergy Clin Immunol. 2011;128(5):948-55. e3.

22. Eichenfield LF, Tom WL, Chamlin SL, Feldman SR, Hanifin JM, Simpson EL, et al. Guidelines of care for the management of atopic dermatitis: section 1 . Diagnosis and assessment of atopic dermatitis. $\mathrm{J} \mathrm{Am}$ Acad Dermatol. 2014;70(2):338-51.

23. Martin R, Nauta A, Ben Amor K, Knippels L, Knol J, Garssen J. Early life: gut microbiota and immune development in infancy. Benef Microbes. 2010;1(4):367-82.

24. Botteman MF, Munasir Z, Sulistomo AW, Horodniceanu EG, Bhanegaonkar AJ, Ji X, et al. Economic value of atopic dermatitis prevention via partially-hydrolyzed whey-based infant formula (PHF-W) use in high-risk, non-exclusively breastfed, Indonesian urban infants: results of a costeffectiveness model. World Nut J. 2019;2(2):43-55.

25. Chua MC, Ben-Amor K, Lay C, Goh AE, Chiang WC, Rao R, et al. Effect of synbiotic on the gut microbiota of cesarean delivered infants: a randomized, double-blind, multicenter study. $J$ Pediatr Gastr Nutr. 2017;65(1):102-6.

26. Abrahamsson TR, Wu RY, Jenmalm MC. Gut microbiota and allergy: the importance of the pregnancy period. Pediatr Res. 2015;77(1-2):214-9.

27. Fox A, Bird JA, Fiocchi A, Knol J, Meyer R, Salminen $\mathrm{S}$, et al. The potential for pre-, pro-and synbiotics in the management of infants at risk of cow's milk allergy or with cow's milk allergy: An exploration of the rationale, available evidence and remaining questions. World Allergy Organ. 2019;12(5):100034.

28. Moya-Pérez A, Luczynski P, Renes IB, Wang S, Borre $\mathrm{Y}$, Anthony Ryan $\mathrm{C}$, et al. Intervention strategies for cesarean section-induced alterations in the microbiota-gut-brain axis. Nutr Rev. 2017;75(4):225-40.

29. Moossavi S, Miliku K, Sepehri S, Khafipour E and Azad MB. The Prebiotic and Probiotic Properties of Human Milk: Implications for Infant Immune Development and Pediatric Asthma. Front Pediatr. 6:197

30. Heikkilä MP, Saris PE. Inhibition of Staphylococcus aureus by the commensal bacteria of human milk. $J$ Appl Microbiol 2003; 95:471-478.

31. Boix-Amorós A, Collado MC, Mira A. Relationship Between Milk Microbiota, Bacterial Load,
Macronutrients, and Human Cells During Lactation. Front Microbiol. 2016;7:492.

32. Jost $T$, Lacroix $C$, Braegger $C$, Chassard $C$. Assessment of Bacterial Diversity in Breast Milk Using Culture-Dependent and Culture-Independent Approaches. Br J Nutr. 2013 Oct;110(7):1253-62.

33. Plaza-Díaz J, Fontana L and Gil A. Human Milk Oligosaccharides and Immune System Development. Nutrients. 2018; 10:1038.

34. Knol J, Scholtens P, Kafka C, Steenbakkers J, Gro S, Helm K, et al. Colon microflora in infants fed formula with galacto-and fructo-oligosaccharides: more like breast-fed infants. J Pediatr Gastr Nutr. 2005;40(1):36-42.

35. Moro, G., et al., A mixture of prebiotic oligosaccharides reduces the incidence of atopic dermatitis during the first six months of age. Archives of Disease in Childhood. 2006. 91(10): p. 814-9.

36. Arslanoglu, S., et al., Early dietary intervention with a mixture of prebiotic oligosaccharides reduces the incidence of allergic manifestations and infections during the first two years of life. J Nutr. 2008. 138(6): p. 1091-5.

37. Arslanoglu, S., et al., Early neutral prebiotic oligosaccharide supplementation reduces the incidence of some allergic manifestations in the first 5 years of life. J Biol Regul Homeost Agents. 2012. 26(3 Suppl): p. 49-59.

38. Ismail IH, Boyle RJ, Licciardi PV, Oppedisano F, Lahtinen S, Robins-Browne RM, et al. Early gut colonization by Bifidobacterium breve and $\mathrm{B}$. catenulatum differentially modulates eczema risk in children at high risk of developing allergic disease. Pediat Allerg Imm-UK. 2016;27(8):838-46.

39. Li N, Yu Y, Chen X, Gao S, Zhang Q, Xu C. Bifidobacterium breve $\mathrm{M}-16 \mathrm{~V}$ alters the gut microbiota to alleviate OVA-induced food allergy through IL-33/ST2 signal pathway. J Cell Physiol. 2020.

40. Fiocchi A, Pawankar R, Cuello-Garcia C, Ahn K, AlHammadi S, Agarwal A, et al. World Allergy Organization-McMaster University Guidelines for Allergic Disease Prevention (GLAD-P): Probiotics

41. Cuello-Garcia CA, Fiocchi A, Pawankar R,YepesNuñez JJ, Morgano GP, Zhang Y, Ahn K, et al. World Allergy Organization-McMaster University Guidelines for Allergic Disease Prevention (GLADP): Prebiotics

42. Hegar B, Wibowo Y, Basrowi RW, Ranuh RG, Sudarmo SM, Munasir Z, et al. The Role of Two Human Milk Oligosaccharides, 2'-Fucosyllactose and Lacto-N-Neotetraose, in Infant Nutrition. Pediatr Gastroenterol Hepatol Nutr. 2019 Jul;22(4):330-40. 